\title{
Effect of combinations of marketed human anthelmintic drugs against Trichuris muris in vitro and in vivo
}

Jennifer Keiser ${ }^{1,2^{*}}$, Lucienne Tritten ${ }^{1,2}$, Roberto Adelfio ${ }^{1,2}$ and Mireille Vargas ${ }^{1,2}$

\begin{abstract}
Background: Soil-transmitted helminth (STH) infections are responsible for a huge public health burden, however treatment options are limited. The discovery and development of novel efficacious drugs or drug combinations for the treatment of STH infections therefore has a high research priority.

Methods: We studied drug combination effects using the main standard anthelmintics, albendazole, mebendazole, levamisole, pyrantel pamoate and ivermectin in the Trichuris muris model. Drug combinations were first tested in vitro and additive and synergistic combinations investigated further in vivo in female mice using ratios based on the $\mathrm{ED}_{50}$ of the respective drugs.

Results: In vitro all 10 combinations of the standard anthelmintics tested against T. muris revealed synergistic behavior. We identified three drug combinations in vivo as strongly synergistic, namely mebendazole-ivermectin (Combination index $(\mathrm{Cl})=0.16)$, mebendazole-levamisole $(\mathrm{Cl}=0.17)$ and albendazole-mebendazole $(\mathrm{Cl}=0.23)$. For albendazole-ivermectin, moderate synergism was observed ( $\mathrm{Cl}=0.81)$ and for albendazole-levamisole a nearly additive effect was documented $(\mathrm{Cl}=0.93)$ in vivo. Five combinations (albendazole-pyrantel pamoate, mebendazole-pyrantel pamoate, levamisole-pyrantel pamoate, levamisole-ivermectin and pyrantel pamoateivermectin) were antagonistic in vivo.
\end{abstract}

Conclusion: Our results strengthen the evidence that combination chemotherapy might play a role in the treatment of Trichuris infections. Albendazole-mebendazole should be studied in greater detail in preclinical studies.

Keywords: Combination chemotherapy, Trichuris muris, Albendazole, Mebendazole, Pyrantel pamoate, Ivermectin, Levamisole, In vitro, In vivo

\section{Background}

Soil-transmitted helminth (STH) infections impose a major public health burden mostly among poor populations. It has been estimated that in 2010, 5.3 billion people, of these 1.0 billion school-aged children, were at risk of infection with at least one STH species [1]. Preventive chemotherapy, with the two benzimidazoles, albendazole and mebendazole forms the bedrock of helminth control initiatives preventing morbidity due to helminthiases [2]. However, the number of drugs used to treat infections with STH are limited, have only a low

\footnotetext{
* Correspondence: jennifer.keiser@unibas.ch

${ }^{1}$ Department of Medical Parasitology and Infection Biology, Swiss Tropical and Public Health Institute, P.O. Box, CH-4002, Basel, Switzerland

2University of Basel, Basel $\mathrm{CH}-4003$, Switzerland
}

efficacy against Trichuris trichiura as a single dose treatment and there is a potential for the development of drug resistance $[3,4]$. Therefore, recently the Disease Reference Group on Helminth Infections established by the Special Programme for Research and Training in Tropical Diseases (TDR) was given the mandate to develop a research and development agenda for intervention tools considered necessary for control and elimination of human helminthiases [5]. The discovery and development of novel efficacious drugs was identified as one of the top research priorities [6]. In addition, it has been emphasized that more research should be undertaken to investigate whether combinations of different anthelmintics would reveal synergistic effects and would therefore improve control of helminth infections

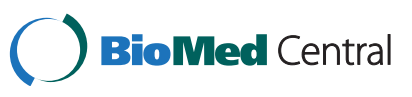


[6]. Indeed, to date, limited research has been done to assess whether combinations of different anthelmintics produce synergistic effects. We have recently studied the effects of oxantel pamoate in combination with standard drugs albendazole, mebendazole, pyrantel pamoate, levamisole and ivermectin against $T$. muris in vitro and in vivo (Keiser et al., submitted for publication). Using Caenorhabditis elegans, significant synergy was documented between Cry proteins from Bacillus thuringiensis and tribendimidine or levamisole [7]. Similarly, tribendimidine combined with levamisole showed synergistic effects against Ancylostoma ceylanicum in vitro and in vivo in male Syrian Golden hamsters [8]. Several clinical trials have also been conducted with drug combinations in patients infected with hookworms or $T$. trichiura [4,9-11], however, the scientific basis for selecting these combinations is not clear. Prior to clinical trials, drug combination studies in vitro and/or in animal models followed by preclinical drug interaction studies should be carried out to determine the basis and rationale for studies in humans and to confirm that concomitant use of the medications is safe.

The aim of the present study was to analyze drug combination effects using the main standard anthelmintics albendazole, mebendazole, levamisole, pyrantel pamoate and ivermectin in the T. muris mouse model. Drug combinations were first tested in vitro and additive and synergistic combinations further investigated in vivo.

\section{Methods}

\section{Drugs}

Albendazole and levamisole were purchased from Fluka (Buchs, Switzerland). Mebendazole, ivermectin and pyrantel pamoate were acquired from Sigma-Aldrich (Buchs, Switzerland). Drug stock solutions of $10 \mathrm{mg} / \mathrm{ml}$ were prepared in 100\% DMSO (Sigma-Aldrich, Buchs, Switzerland) for in vitro studies. These were stored at $4^{\circ} \mathrm{C}$ pending use. For in vivo treatments, the drugs were suspended in $10 \%$ Tween 80 [70\% EtOH (70:30 v/v)] (Buchs, Switzerland) and $90 \%$ deionised $\mathrm{H}_{2} \mathrm{O}$ just prior to oral administration.

\section{Ethics statement}

Experiments were performed in accordance with the $3 \mathrm{R}$ rules for animal experiments. The current study was approved by the cantonal veterinary office Basel-Stadt (Switzerland) based on Swiss cantonal and national regulations (permission no. 2070).

\section{Animals and infections}

Four week-old female C57BL/10 mice were purchased from Harlan (Blackthorn, UK). Before infection, animals were acclimatized for one week in the animal facility. Groups of 10 mice were kept in macrolon cages with ad libitum access to water and rodent food (Rodent Blox from Eberle NAFAG, Gossau, Switzerland). The T. muris life-cycle and maintenance have been described elsewhere [12,13]. Briefly, mice were treated with $1 \mathrm{mg} / \mathrm{l}$ dexamethasone (dexamethasone-water soluble, SigmaAldrich) supplied in the drinking water 2 days before infection by oral gavage with 200 embryonated T. muris eggs until 2 days preceding treatment. Adult worms were recovered from the intestines of sacrificed animals from day 35 p.i. onwards (binocular, magnification 16x) and kept at $37^{\circ} \mathrm{C}, 5 \% \mathrm{CO}_{2}$, in RPMI medium [10.44 g RPMI 1640 (Gibco, Basel, Switzerland) 5 g albumax $\mathrm{H}$ (Gibco), 5.94 g HEPES (Sigma-Aldrich) and $2.1 \mathrm{~g}$ sodium bicarbonate (Sigma-Aldrich) in $1 \mathrm{l} \mathrm{dH}_{2} \mathrm{O}$ ] supplemented with $5 \% \mathrm{v} / \mathrm{v}$ amphotericin B (stock: $250 \mu \mathrm{g} / \mathrm{ml}$, Sigma-Aldrich) and $1 \% \mathrm{v} / \mathrm{v}$ penicillin-streptomycin (stock: $10,000 \mathrm{U} / \mathrm{ml}$ penicillin $+10 \mathrm{mg} / \mathrm{ml}$ streptomycin, Sigma-Aldrich).

\section{In vitro drug combination studies}

Two adult worms were incubated in each well of a 48 well plate containing $500 \mu \mathrm{l}$ pre-warmed supplemented RPMI medium (described above), and $500 \mu \mathrm{l}$ drug solution. $\mathrm{IC}_{50}$ (50\% inhibitory concentration) values of the standard drugs were determined in our laboratory prior to this work and are summarized in Table 1. In more detail, for levamisole and pyrantel pamoate, $\mathrm{IC}_{50} \mathrm{~s}$ of 16.5

\begin{tabular}{|c|c|}
\hline \multicolumn{2}{|c|}{ Drug activity against $T$. muris adult worms in vitro } \\
\hline Single drug & $\mathrm{IC}_{50} \mathrm{~s}(\mu \mathrm{g} / \mathrm{ml})(\mathrm{r})$ \\
\hline Albendazole & $>200(0.40)^{\S}$ \\
\hline Mebendazole & $>200(0.58)$ \\
\hline Levamisole & $16.5(1.0)^{\S}$ \\
\hline Pyrantel pamoate & $34.1(0.99)^{\S}$ \\
\hline Ivermectin & $>200(0.40)$ \\
\hline Drug combination & $\mathrm{Cl}$ at $\mathrm{IC}_{50}$ \\
\hline Albendazole-mebendazole & $<0.001$ \\
\hline Albendazole-levamisole & 0.12 \\
\hline Albendazole-pyrantel pamoate & 0.11 \\
\hline Albendazole-ivermectin & $<0.001$ \\
\hline Mebendazole-levamisole & 0.04 \\
\hline Mebendazole-pyrantel pamoate & 0.12 \\
\hline Mebendazole-ivermectin & $<0.001$ \\
\hline Levamisole-pyrantel pamoate & 0.007 \\
\hline Levamisole-ivermectin & 0.12 \\
\hline Pyrantel pamoate-ivermectin & 0.14 \\
\hline
\end{tabular}

$\mathrm{IC}_{50}$ median inhibitory concentration. $\mathrm{r}$ = linear correlation coefficient of the median-effect plot, indicating the goodness of fit. $r \geq 0.85$ indicates a satisfactory fit. ${ }^{\S} \mathrm{IC}_{50}$ s of albendazole, levamisole, and pyrantel pamoate have been published elsewhere [13]. 
and $34.1 \mu \mathrm{g} / \mathrm{ml}$ were reported [13]. If no activity of the drugs on the worms was observed at the highest concentration tested as for albendazole, mebendazole and ivermectin, $\mathrm{IC}_{50}$ values of $200 \mu \mathrm{g} / \mathrm{ml}$ were chosen arbitrarily for the drug combination studies presented here. Six concentrations of each drug concentration were tested, starting with the $\mathrm{IC}_{50}$ value of each partner drug $\left(\mathrm{IC}_{50}+\mathrm{IC}_{50}\right)$, which was two-fold diluted up to a final concentration of $1 / 32 \mathrm{IC}_{50}+1 / 32 \mathrm{IC}_{50}$. Control worms were incubated in medium with equivalent DMSO concentrations (maximum $4 \% \mathrm{v} / \mathrm{v}$ ). The plate was incubated at $37^{\circ} \mathrm{C}$ and $5 \% \mathrm{CO}_{2}$. After $72 \mathrm{~h}$ the motility was evaluated under a light microscope (magnification 20-80x) using a motility scale from 0 to $3(0=$ dead, $1=$ very low motility, 2 = low motility, 3 = normal motility) as described previously [13]. Experiments were carried out in duplicate and repeated at least twice. Data obtained from the individual experiments were averaged and adjusted to the values obtained from untreated controls. The nature of each drug combination was characterized by a combination index (CI) as described by Chou [14] and calculated with CompuSyn (CompuSyn, version 3.0.1, ComboSyn, Inc., Paramus, NJ 2007). We used the following classification $\mathrm{CI}<0.1$ very strong synergism, $\mathrm{CI}$ : $0.1-0.3$ strong synergism, CI: 0.3-0.7 synergism, CI: 0.7-0.85 moderate synergism, CI: 0.85-0.9 slight synergism, CI: 0.9-1.1 nearly additive and $\mathrm{CI}>1.1$ antagonism. Synergistic and additive drug combinations identified in vitro $(\mathrm{CI} \leq 1)$ were tested in infected animals using a constant dose ratio as described below.

\section{In vivo drug combination studies}

On day 40 post-infection the presence of a chronic T. muris infection was confirmed in each mouse by an egg positive stool examination. Groups of 4 mice were assigned to treatment or served as untreated control. $\mathrm{ED}_{50} \mathrm{~S}$ (median effective doses) of each standard drug had been determined in our laboratory prior to this work and were as follows; $345 \mathrm{mg} / \mathrm{kg}$ for albendazole, $79 \mathrm{mg} / \mathrm{kg}$ for mebendazole (Keiser et al., submitted for publication), 46 $\mathrm{mg} / \mathrm{kg}$ for levamisole and $4 \mathrm{mg} / \mathrm{kg}$ for ivermectin [15]. An arbitrary value of $300 \mathrm{mg} / \mathrm{kg}$ was chosen for pyrantel pamoate, for which no $\mathrm{ED}_{50}$ could be calculated. The ratio of the $\mathrm{ED}_{50} \mathrm{~S}$ of each partner drug was chosen as starting dose (drug $1 \mathrm{ED}_{50^{+}}$drug $2 \mathrm{ED}_{50}$ ). If a combination treatment decreased the worm burden by at least 75\% (threshold for additivity when the dose effect curves for both drugs are hyperbolic [14]), the drug doses were reduced by half. Stools were collected for up to $72 \mathrm{~h}$ post-treatment and the expelled worms were counted. Mice were killed one week post-treatment by exposure to $\mathrm{CO}_{2}$, the entire intestine was dissected and all remaining worms counted. Drug activity was expressed by worm burden reduction (WBR) and worm expulsion rate (WER) as described elsewhere [8].
The significance of the WBR was tested using the KruskalWallis test (several treatment doses vs. controls) or the Mann-Whitney U-test (one treatment dose vs. control) using StatsDirect (version 2.4.5; StatsDirect Ltd; Cheshire, $\mathrm{UK})$. Combination indices (CI) were calculated with CompuSyn as described above.

\section{Results}

\section{Activity of combinations of standard anthelmintics} against $T$. muris in vitro

All 10 combinations of the standard anthelmintics tested against $T$. muris in vitro resulted in a synergistic interaction (Table 1$)$. A very strong synergism $(\mathrm{CI}<0.1)$ was observed for albendazole-mebendazole, albendazoleivermectin and mebendazole-ivermectin (all $\mathrm{CIs}<0.001$ ), mebendazole-levamisole $(\mathrm{CI}=0.04)$ and levamisole-pyrantel pamoate $(\mathrm{CI}=0.007)$. For the other 5 combinations (albendazole-levamisole, albendazole-pyrantel pamoate, mebendazole-pyrantel pamoate, levamisole-ivermectin and pyrantel pamoate-ivermectin a strong synergism (CI=0.11-0.14) was determined.

\section{Activity of combinations of standard anthelmintics against $T$. muris in vivo}

Since all anthelmintic drug combinations yielded synergistic effects in vitro, they were further investigated in vivo using the $\mathrm{ED}_{50}$ of each drug (drug $1 \mathrm{ED}_{50}+$ drug $2 \mathrm{ED}_{50}$ ) as starting dose. The results are summarized in Table 2. We identified three drug combinations as strongly synergistic, namely mebendazole-ivermectin $(\mathrm{CI}=0.16)$, mebendazole-levamisole $(\mathrm{CI}=0.17)$ and albendazole-mebendazole $(\mathrm{CI}=0.23)$. These drug combinations still achieved significant WBRs when drug dosages of $1 / 16 \mathrm{ED}_{50}$ were combined. For example, a WBR of $70.3 \%$ was achieved, when $4.9 \mathrm{mg} / \mathrm{kg}$ mebendazole was combined with $0.25 \mathrm{mg} / \mathrm{kg}$ ivermectin. Note that mebendazole plus ivermectin (at the highest dose) was the only combination that resulted in complete elimination of worms.

For albendazole plus ivermectin a moderate synergism was observed $(\mathrm{CI}=0.81)$ and when albendazole was administered together with levamisole a nearly additive effect was documented $(\mathrm{CI}=0.93)$.

Five combinations (albendazole-pyrantel pamoate, mebendazole-pyrantel pamoate, levamisole-pyrantel pamoate, levamisole-ivermectin and pyrantel pamoateivermectin) revealed an antagonistic behavior. Four of these drug combinations (mebendazole-pyrantel pamoate, levamisole-pyrantel pamoate, levamisole-ivermectin and pyrantel pamoate-ivermectin) showed an effect at the highest dosage tested $\left(\mathrm{ED}_{50}+\mathrm{ED}_{50}\right)$ with WBRs of 79.7-96.8\% but only low activity when half of these dosages were used. Albendazole-pyrantel pamoate $\left(E D_{50}+E_{50}\right)$ resulted in only a moderate worm burden reduction of $44.2 \%$. 
Table 2 Worm expulsion rates, worm burden reductions and corresponding combination index values obtained following treatment of mice harboring adult $T$. muris with combinations of standard anthelmintics

\begin{tabular}{|c|c|c|c|c|c|c|c|}
\hline Group & Dose $(\mathrm{mg} / \mathrm{kg})$ & $\begin{array}{l}\text { Mean number of } \\
\text { worms (SD) }\end{array}$ & $\begin{array}{l}\text { Mean number of } \\
\text { expelled worms (SD) }\end{array}$ & $\begin{array}{l}\text { Worm expulsion } \\
\text { rate }(\%)\end{array}$ & $\begin{array}{l}\text { Worm burden } \\
\text { reduction (\%) }\end{array}$ & $P$-value & $\begin{array}{l}\text { Combination } \\
\text { index at } \mathrm{ED}_{50} \\
\end{array}$ \\
\hline Control 1 & - & $93.25(9.46)$ & $0.75(0.96)$ & 0.8 & - & - & - \\
\hline Control 2 & - & $123.25(35.07)$ & $0.25(0.50)$ & 0.2 & - & - & - \\
\hline Control 3 & - & 91.25 (23.73) & $0(0)$ & 0 & - & - & - \\
\hline Control 4 & - & 78.33 (20.79) & $0(0)$ & 0 & - & - & - \\
\hline Control 5 & - & $85.71(21.75)$ & $0(0)$ & 0 & - & - & - \\
\hline Control 6 & - & 82.57 (30.59) & $0(0)$ & 0 & - & - & - \\
\hline Control 7 & - & $94.4(39.21)$ & $0(0)$ & 0 & - & - & - \\
\hline Control 8 & - & $56.50(22.07)$ & $0(0)$ & 0 & - & - & - \\
\hline \multirow[t]{4}{*}{ ABZ-MBZ } & $345+79^{3}$ & $77.50(63.07)$ & $60.50(55.87)$ & 78.06 & 81.37 & $<0.001^{\mathrm{a}}$ & 0.23 \\
\hline & $172.5+39.5^{4}$ & $73.25(102.66)$ & $62.75(91.34)$ & 85.67 & 86.60 & & \\
\hline & $86.25+19.75^{7}$ & $95.0(5.66)$ & $63.0(1.41)$ & 66.32 & 66.10 & & \\
\hline & $43.125+9.875^{8}$ & 77.75 (33.54) & $47.75(22.34)$ & 61.41 & 46.90 & & \\
\hline \multirow[t]{2}{*}{ ABZ-LEV } & $345+46^{5}$ & $117.50(112.86)$ & $107.0(112.75)$ & 91.06 & 87.75 & $0.001^{\mathrm{a}}$ & 0.93 \\
\hline & $172.5+23^{7}$ & $89.25(42.0)$ & $46.50(35.61)$ & 52.10 & 54.71 & & \\
\hline ABZ-PYR & $345+300^{4}$ & $63.5(41.30)$ & $19.75(11.76)$ & 31.10 & 44.15 & $0.229^{b}$ & - \\
\hline \multirow[t]{2}{*}{ ABZ-IVM } & $345+4^{2}$ & $136.50(14.98)$ & $109.50(15.76)$ & 80.22 & 78.05 & $0.003^{\mathrm{a}}$ & 0.81 \\
\hline & $172.5+2^{7}$ & $80.50(17.06)$ & $41.50(9.26)$ & 51.55 & 58.69 & & \\
\hline \multirow[t]{5}{*}{ MBZ-LEV } & $79+46^{1}$ & $86.0(53.20)$ & $82.75(48.11)$ & 96.22 & 96.49 & $<0.001^{a}$ & 0.17 \\
\hline & $39.5+23^{2}$ & $82.0(17.87)$ & $74.5(21.32)$ & 90.85 & 93.90 & & \\
\hline & $19.75+12.5^{3}$ & $96.25(37.25)$ & $81.25(39.10)$ & 84.42 & 83.56 & & \\
\hline & $9.875+6.25^{4}$ & $61.25(33.98)$ & $50.50(37.36)$ & 82.45 & 86.28 & & \\
\hline & $4.94+3.125^{7}$ & $125.50(71.01)$ & $48.75(37.55)$ & 38.84 & 18.70 & & \\
\hline \multirow[t]{2}{*}{ MBZ-PYR } & $79+300^{4}$ & $41.25(24.65)$ & $38.75(24.92)$ & 93.94 & 96.81 & $0.049^{a}$ & N.D. \\
\hline & $39.5+150^{8}$ & $161.75(98.22)$ & $113.75(77.82)$ & 70.32 & 15.04 & & \\
\hline \multirow[t]{6}{*}{ MBZ-IVM } & $79+4^{2}$ & $92.50(26.71)$ & $92.50(26.71)$ & 100 & 100 & $<0.001^{\mathrm{a}}$ & 0.16 \\
\hline & $39.5+2^{2}$ & 70.33 (32.04) & $68.0(32.19)$ & 96.68 & 98.10 & & \\
\hline & $19.75+1^{3}$ & 46.25 (18.23) & $36.75(11.53)$ & 79.46 & 89.59 & & \\
\hline & $9.875+0.5^{4}$ & $54.0(44.74)$ & $41.50(36.74)$ & 76.85 & 84.04 & & \\
\hline & $4.94+0.25^{7}$ & $101.0(57.30)$ & $73.0(34.70)$ & 72.28 & 70.34 & & \\
\hline & $2.47+0.125^{8}$ & $70.33(26.86)$ & $16.67(22.85)$ & 23.70 & 5.01 & & \\
\hline \multirow[t]{2}{*}{ LEV-PYR } & $46+300^{6}$ & $46.25(18.84)$ & $29.50(19.28)$ & 63.78 & 79.71 & $0.057^{\mathrm{a}}$ & N.D. \\
\hline & $23+150^{8}$ & $87.75(57.34)$ & $10.50(10.88)$ & 11.97 & 0 & & \\
\hline \multirow[t]{2}{*}{ LEV-IVM } & $46+4^{4}$ & $66.25(42.84)$ & $59.0(42.78)$ & 89.06 & 90.74 & $0.009^{a}$ & 1.38 \\
\hline & $23+2^{8}$ & $60.0(24.51)$ & $14.50(13.80)$ & 24.17 & 19.47 & & \\
\hline \multirow[t]{2}{*}{ PYR-IVM } & $300+4^{4}$ & $52.25(36.04)$ & $46.75(32.71)$ & 89.47 & 92.98 & $0.032^{\mathrm{a}}$ & N.D. \\
\hline & $150+2^{8}$ & $122.50(112.38)$ & $32.75(17.39)$ & 26.73 & 0 & & \\
\hline
\end{tabular}

Numbers in superscript refer to the corresponding control group. ${ }^{\mathrm{a}} P$-values were obtained from the Kruskal-Wallis test (several treatment doses vs. controls), ${ }^{\mathrm{b}} P$ values were obtained from the Mann-Whitney $\mathrm{U}$ test (one treatment dose vs. controls). The $\mathrm{Cl}$ at $\mathrm{ED}_{50}$ are based on worm burden reductions. N.D. $=$ not determined. ABZ: albendazole; MBZ: mebendazole; LEV: levamisole; PYR: pyrantel pamoate; IVM: ivermectin.

\section{Discussion}

The treatment of T. trichiura infections in humans has significant deficiencies, since none of the available anthelmintics achieves high cure rates and egg reduction rates, when the drugs are administered as single doses in the framework of preventive chemotherapy treatment campaigns $[2,3]$. Combination chemotherapy is common medical practice in several medical fields such as cancer, bacterial infections, HIV or malaria $[4,16,17]$ as well as in veterinary medicine [18] since an efficacious intervention 
often requires the combined action of two or more interacting therapeutic components. Furthermore, avoiding drug resistance has been a major rationale for combination chemotherapy from the start $[16,17]$.

To the best of our knowledge, we have for the first time thoroughly evaluated the activity of combinations of available marketed human anthelmintics against the laboratory model T. muris in vitro and in vivo. In vitro, all combinations were found to be synergistic (Figure 1) and therefore followed up in vivo. Four drug combinations were identified in vivo using drug doses based on $\mathrm{ED}_{50}$ values, which showed synergistic properties namely mebendazole-ivermectin, mebendazole-levamisole, albendazole-mebendazole and albendazole plus ivermectin. In addition, for the combination of albendazole and levamisole an additive trichuricidal effect was documented (Figure 1). A good correlation between the in vitro and in vivo results was observed with the 3 drug combinations showing a strongly synergistic effect

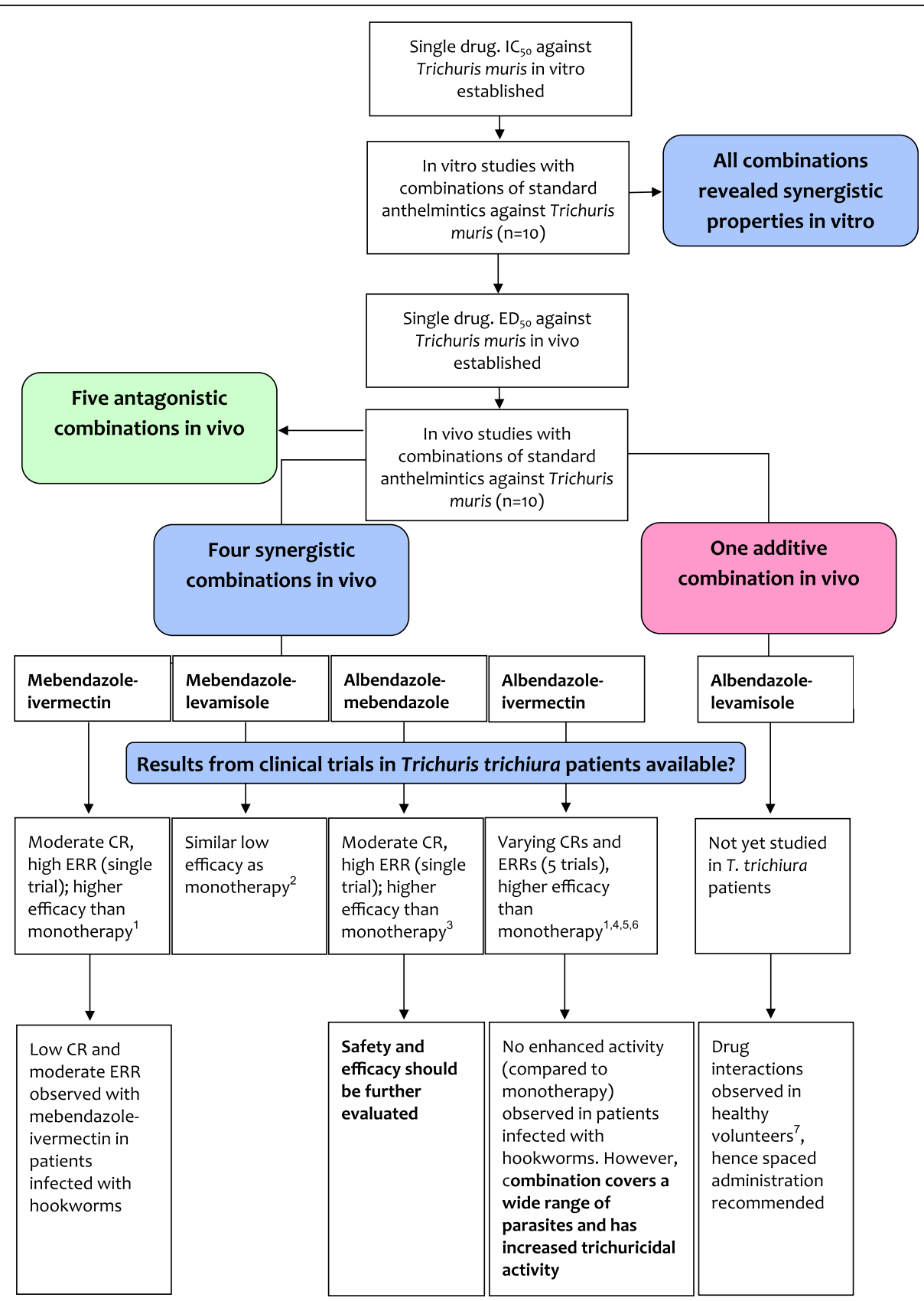

Figure 1 Study flow diagram, illustrating the results of our studies and placing them in context with clinical trials. ': Reference [10], ${ }^{2}$ Reference [22], ${ }^{3}$ Reference [11], ${ }^{4}$ Reference [24], ${ }^{5}$ Reference [25], ${ }^{6}$ Reference [26], ${ }^{7}$ Reference [27]. ERR: egg reduction rate; CR: cure rate. 
in vitro (albendazole-mebendazole, albendazole-ivermectin and mebendazole-ivermectin) also revealing synergism in vivo. It is interesting to note that the synergistic combinations identified comprise at least one benzimidazole. Drug combinations containing pyrantel pamoate as well as levamisole combined with ivermectin were found to be antagonistic. An increased bioavailability of ivermectin was observed when the drug was given concurrently with levamisole in healthy volunteers [19], however, it is not clear whether this pharmacokinetic interaction has an influence on the activity against T. muris in mice. Unfortunately, possible mechanisms underlying synergistic or antagonistic effects are presently not available for any of these anthelmintic drug combinations. It has recently been suggested that an increased efficacy of an albendazole-mebendazole combination over albendazole or mebendazole monotherapy might be explained by an extended contact period of the worms with the active compounds (active compounds present successively: mebendazole first followed by the main metabolite of albendazole, albendazole sulfoxide [11]). However, it has been suggested that the parent drug albendazole, more lipophilic, exerts the main anthelmintic action as the transcuticular diffusion is thought to be more important for drug uptake by worms [20,21], which would contradict the above mentioned hypothesis of an increased activity due to albendazole sulfoxide.

Clinical trials have been conducted with the four synergistic combinations reported here (Figure 1). Ironically these trials have been conducted without any preclinical supporting information, either on efficacy or potential toxicity. No enhanced trichuricidal efficacy was observed when a combination of mebendazole and levamisole was given to school-aged children on Pemba island [22]. However, our findings of the synergistic effect of albendazole-ivermectin, mebendazole-ivermectin and albendazole-mebendazole in T. muris-infected mice are in line with results from patients infected with $T$. trichiura. Briefly, five studies investigated the efficacy of albendazoleivermectin [10,23-26] and increased cure rates and egg reduction rates for the combination compared to albendazole or ivermectin were observed in all trials. Clearly, a tremendous advantage of an albendazole-ivermectin combination is its broad spectrum of activity targeting not only the soiltransmitted helminths, including Strongyloides stercoralis but also lymphatic filariasis and onchocerciasis. In three of the trials the effect of albendazole-ivermectin was also assessed against infections with hookworms, however, no increased therapeutic benefit was observed for albendazoleivermectin over albendazole [10,22,24]. Moreover, the combination has already been studied in onchocerciasis patients and no pharmacokinetic interactions were observed [19]. Mebendazole-ivermectin showed an even superior activity to albendazole-ivermectin [10], which might be explained by the higher sensitivity of Trichuris spp. to mebendazole than albendazole. However, it is important to mention that the combination of mebendazole and ivermectin only achieved moderate egg reduction rates and low cure rates against hookworm infections [10]. Finally, albendazolemebendazole achieved a higher cure rate $(46.1 \%)$ compared to albendazole (6.0\%) and mebendazole (11\%) in schoolaged children infected with T. trichiura in Uganda [11].

In the present work an additive effect was observed when levamisole was administered in combination with albendazole to T. muris-infected mice. To our knowledge, this combination has not been used in the treatment of trichuriasis. However, Awadzi and colleagues have shown that clinically significant drug interactions occur between the two drugs, resulting in a reduction of albendazole sulfoxide and levamisole plasma levels as well as unexpected adverse events in onchocerciasis patients [27]. Hence, given these pharmacokinetic interactions a simultaneous co-administration of albendazole and levamisole cannot be recommended.

\section{Conclusions}

We have identified four synergistic and one additive combination against $T$. muris in the mouse model. To shed light on the next steps to be undertaken we have placed our findings in context with Phase I and Phase II clinical trials conducted with these drug combinations in previous years. In addition, it has to be emphasized that the four species of nematodes (Ascaris lumbricoides, T. trichiura and both hookworm species, Ancylostoma duodenale and Necator americanus) are treated collectively in preventive chemotherapy programs and the treatments should therefore have high activities against all these species. Albendazole-mebendazole has emerged as an interesting and unexpected drug combination, hence this treatment should be studied in greater detail including drug interaction studies as well as studies against hookworms. In addition, albendazole-ivermectin offers the advantage that it is well studied, has increased trichuricidal activity over albendazole and ivermectin alone and has a broad spectrum of activity.

\section{Competing interests}

The authors declare that they have no competing interests.

\section{Authors' contributions}

JK and LT designed the studies. MV and RA carried out the experiments. JK and LT wrote the manuscript. All authors read and approved the final version of the manuscript.

Acknowledgments

We are grateful to SNSF (project no. PPOOA3-114941 and PPOOP3_135170 to JK) and the Medicor Foundation for financial support.

Received: 19 October 2012 Accepted: 7 December 2012

Published: 11 December 2012 


\section{References}

1. Pullan RL, Brooker SJ: The global limits and population at risk of soiltransmitted helminth infections in 2010. Parasit Vectors 2012, 5:81.

2. WHO: Preventive chemotherapy in human helminthiasis: coordinated use of anthelminthic drugs in control interventions: a manual for health professionals and programme managers. Geneva: World Health Organization; 2006.

3. Keiser J, Utzinger J: Efficacy of current drugs against soil-transmitted helminth infections: systematic review and meta-analysis. JAMA 2008, 299:1937-1948.

4. Keiser J, Utzinger J: The drugs we have and the drugs we need against major helminth infections. Adv Parasitol 2010, 73:197-230.

5. Utzinger J: A research and development agenda for the control and elimination of human helminthiases. PLOS Negl Trop Dis 2012, 6:e1646.

6. Prichard RK, Basanez MG, Boatin BA, McCarthy JS, Garcia HH, Yang GJ, Sripa $B$, Lustigman S: A research agenda for helminth diseases of humans: intervention for control and elimination. PLOS Negl Trop Dis 2012, 6:e1549.

7. Hu Y, Platzer EG, Bellier A, Aroian RV: Discovery of a highly synergistic anthelmintic combination that shows mutual hypersusceptibility. Proc Natl Acad Sci USA 2010, 107:5955-5960.

8. Tritten L, Nwosu U, Vargas M, Keiser J: In vitro and in vivo efficacy of tribendimidine and its metabolites alone and in combination against the hookworms Heligmosomoides bakeri and Ancylostoma ceylanicum. Acta Trop 2012, 122:101-107.

9. Geary TG, Woo K, McCarthy JS, Mackenzie CD, Horton J, Prichard RK, de Silva NR, Olliaro PL, Lazdins-Helds JK, Engels DA, et al: Unresolved issues in anthelmintic pharmacology for helminthiases of humans. Int J Parasitol 2010, 40:1-13.

10. Knopp S, Mohammed KA, Speich B, Hattendorf J, Khamis IS, Khamis AN, Stothard JR, Rollinson D, Marti H, Utzinger J: Albendazole and mebendazole administered alone or in combination with ivermectin against Trichuris trichiura: a randomized controlled trial. Clin Infect Dis 2011, 51:1420-1428.

11. Namwanje $H$, Kabatereine NB, Olsen A: Efficacy of single and double doses of albendazole and mebendazole alone and in combination in the treatment of Trichuris trichiura in school-age children in Uganda. Trans $R$ Soc Trop Med Hyg 2011, 105:586-590.

12. Wakelin D: The stimulation of immunity and the induction of unresponsiveness to Trichuris muris in various strains of laboratory mice. Z Parasitenkd 1970, 35:162-168.

13. Tritten $L$, Silbereisen $A$, Keiser J: In vitro and in vivo efficacy of monepantel (AAD 1566) against laboratory models of human intestinal nematode infections. PLOS Negl Trop Dis 2011, 5:e1457.

14. Chou TC: Drug combination studies and their synergy quantification using the Chou-Talalay method. Cancer Res 2010, 70:440-446.

15. Tritten $L$, Silbereisen $A$, Keiser J: Nitazoxanide: In vitro and in vivo drug effects against Trichuris muris and Ancylostoma ceylanicum, alone or in combination. Int J Parasitol: Drugs and Drug Resistance 2012, 2:98-105.

16. White N: Antimalarial drug resistance and combination chemotherapy. Philos Trans R Soc Lond B Biol Sci 1999, 354:739-749.

17. Jia J, Zhu F, Ma X, Cao Z, Li Y, Chen YZ: Mechanisms of drug combinations: interaction and network perspectives. Nat Rev Drug Discov 2009, 8:111-128.

18. Bartram DJ, Leathwick DM, Taylor MA, Geurden T, Maeder SJ: The role of combination anthelmintic formulations in the sustainable control of sheep nematodes. Vet Parasitol 2012, 186:151-158.

19. Awadzi K, Edwards G, Duke BO, Opoku NO, Attah SK, Addy ET, Ardrey AE, Quartey BT: The co-administration of ivermectin and albendazole-safety, pharmacokinetics and efficacy against Onchocerca volvulus. Ann Trop Med Parasitol 2003, 97:165-178.

20. Alvarez LI, Mottier ML, Sanchez SF, Lanusse CE: Ex vivo diffusion of albendazole and its sulfoxide metabolite into Ascaris suum and Fasciola hepatica. Parasitol Res 2001, 87:929-934.

21. Geary TG, Sangster NC, Thompson DP: Frontiers in anthelmintic pharmacology. Vet Parasitol 1999, 84:275-295.

22. Albonico M, Bickle Q, Ramsan M, Montresor A, Savioli L, Taylor M: Efficacy of mebendazole and levamisole alone or in combination against intestinal nematode infections after repeated targeted mebendazole treatment in Zanzibar. Bull World Health Organ 2003, 81:343-352.

23. Ndyomugyenyi R, Kabatereine N, Olsen A, Magnussen P: Efficacy of ivermectin and albendazole alone and in combination for treatment of soil-transmitted helminths in pregnancy and adverse events: a randomized open label controlled intervention trial in Masindi district, western Uganda. AmJTrop Med Hyg 2008, 79:856-863.

24. Beach MJ, Streit TG, Addiss DG, Prospere R, Roberts JM, Lammie PJ: Assessment of combined ivermectin and albendazole for treatment of intestinal helminth and Wuchereria bancrofti infections in Haitian schoolchildren. AmJTrop Med Hyg 1999, 60:479-486.

25. Ismail MM, Jayakody RL: Efficacy of albendazole and its combinations with ivermectin or diethylcarbamazine (DEC) in the treatment of Trichuris trichiura infections in Sri Lanka. Ann Trop Med Parasitol 1999, 93:501-504.

26. Belizario VY, Amarillo ME, De Leon WU, Reyes AE DI, Bugayong MG, Macatangay BJC: A comparison of the efficacy of single doses of albendazole, ivermectin, and diethylcarbamazine alone or in combinations against Ascaris and Trichuris spp. Bull World Health Organ 2003, 81:35-42.

27. Awadzi K, Edwards G, Opoku NO, Ardrey AE, Favager S, Addy ET, Attah SK, Yamuah LK, Quartey BT: The safety, tolerability and pharmacokinetics of levamisole alone, levamisole plus ivermectin, and levamisole plus albendazole, and their efficacy against Onchocerca volvulus. Ann Trop Med Parasitol 2004, 98:595-614.

doi:10.1186/1756-3305-5-292

Cite this article as: Keiser et al:: Effect of combinations of marketed human anthelmintic drugs against Trichuris muris in vitro and in vivo. Parasites \& Vectors 2012 5:292.

\section{Submit your next manuscript to BioMed Central and take full advantage of:}

- Convenient online submission

- Thorough peer review

- No space constraints or color figure charges

- Immediate publication on acceptance

- Inclusion in PubMed, CAS, Scopus and Google Scholar

- Research which is freely available for redistribution

Submit your manuscript at www.biomedcentral.com/submit
C BioMed Central 